\title{
Spontaneous perirenal hemorrhage in a pregnant woman due to the rupture of a simple renal cyst
}

\author{
Saeed Aali ${ }^{*}$, Fatemeh Moradi ${ }^{2}$ \\ 'Department of Urology, Shahrekord University of Medical Sciences, Shahrekord, Iran \\ ${ }^{2}$ Department of Community Nutrition, School of Nutrition and Food Science, Isfahan University of Medical Sciences, Isfahan, Iran
}

*Corresponding Author: Saeed Aali, Clinical Research Development Unit, Ayatollah Kashani Hospital, Parastar Street, Shahrekord, Iran. Phone: +989132082196, Email: saeedaali1350@gmail.com

\begin{abstract}
Renal cysts are diagnosed by ultrasonography, computerized tomography (CT), or urography. A 36-year-old pregnant woman (G2, Ab1, within the gestational age of 38 weeks) with upper abdominal pain starting from two hours ago, vomiting, and nausea was referred to the emergency department. Although abdominal examinations revealed costovertebral angle tenderness, there were no signs of peritoneal irritation, and all vital signs of the patient were in a normal range. Initial investigation results including blood count (Hb: 11.4), BUN/Cr, $\mathrm{Na} / \mathrm{K}$, and UA were normal. She underwent a cesarean section (c/s) due to reduced fetal heart rate (FHR). After C/S,the CT scan showed a large left retroperitoneal hematoma with an anterosuperior displacement of the kidney and active bleeding in the cystic lesion of the upper pole of the kidney. Pathological examinations also confirmed a simple cyst. Overall, simple renal cysts should be considered as a rare cause of spontaneous perinatal hemorrhage (SPH) in pregnancy. The diagnosis and treatment of SPH in pregnancy are also critical challenges. Keywords: Renal cyst, Pregnancy, Spontaneous perinatal hemorrhage
\end{abstract}

Received: May 26 2021, Accepted: December 1 2021, ePublished: December 62021

\section{Introduction}

Simple cysts are the most common cystic lesions that are prevalent in the human kidneys. Cysts are typically discovered incidentally on ultrasonography, computerized tomography (CT), or urography in children and adults. However, cysts can produce an abdominal mass or pain, hematuria secondary to rupture into the pyelocalyceal system, and hypertension secondary to segmental ischemia (1). Spontaneous massive hemorrhage into a cyst (bleeding without trauma) is a rare condition (2). This study reported a 36-year-old pregnant woman with a spontaneous massive hemorrhage of the left renal cyst.

\section{Case Presentation}

A 36-year-old pregnant woman (G2, Ab1, in the gestational age of 38 weeks) was referred to the emergency department with left flank pain starting two hours ago.

The pain had suddenly started and was constant, and then radiated into the upper abdomen and was accompanied by nausea and vomiting.

The patient had no urinary symptoms but was oliguric.On physical examination, besides, she showed a temperature rate of $36.3^{\circ} \mathrm{C}$ (oral), the pulse rate of $100 /$ minute, respiratory rate of 18 breathe per minutes, blood pressure of 100 systolic to 70 diastolic $\mathrm{mm} \mathrm{Hg}$, and the fetal heart rate (FHR) of 140. Abdominal examinations revealed costovertebral angle tenderness although there were no signs of peritoneal irritation. Initial tests including blood count (Hb: 11.4), BUN/Cr, $\mathrm{Na} / \mathrm{K}$, and UA were in the normal range.

Abdominal ultrasonography showed severe left hydronephrosis and free fluid in the abdomen. The patient gradually lost hemoglobin and FHR and simultaneously received fluid and blood. She was transferred to the operating room and underwent a cesarean section.

During surgery, a large non-pulsatile hematoma was observed in the left retroperitoneal space with a slight leaky of blood into the peritoneal space. Due to severe hydronephrosis and oliguria, the patient underwent cystoscopy and bilateral double J placement. The abdomen was then closed, and a CT scan of the abdomen and pelvis was performed with intravenous contrast, representing a large left retroperitoneal hematoma with an anterosuperior displacement of the kidney and active bleeding from a cystic lesion of the upper pole of the kidney (Figures 1 and 2). In laparotomy and retroperitoneal exploration, active bleeding from the cyst at the upper pole was observed, and then a partial nephrectomy was performed as well. The pathological examination also confirmed a simple cyst (Figure 3 ).

\section{Discussion}

This report focused on describing a 36-year pregnant woman with a hemorrhagic renal cyst in 38 weeks of

(C) 2021 The Author(s); Published by Shahrekord University of Medical Sciences. This is an open-access article distributed under the terms of the Creative Commons Attribution License (http://creativecommons.org/licenses/by/4.0), which permits unrestricted use, distribution, and reproduction in any medium, provided the original work is properly cited. 


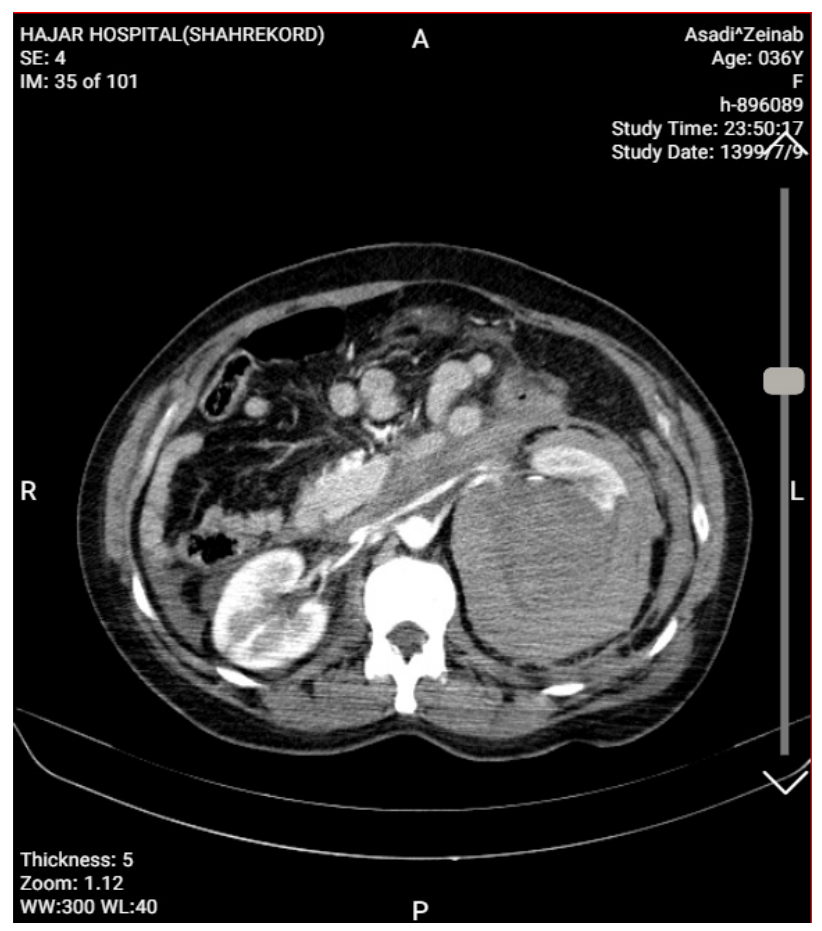

Figure 1. CT scan indicating the anterosuperior displacement of the left kidney. Note. CT: Computerized tomography.

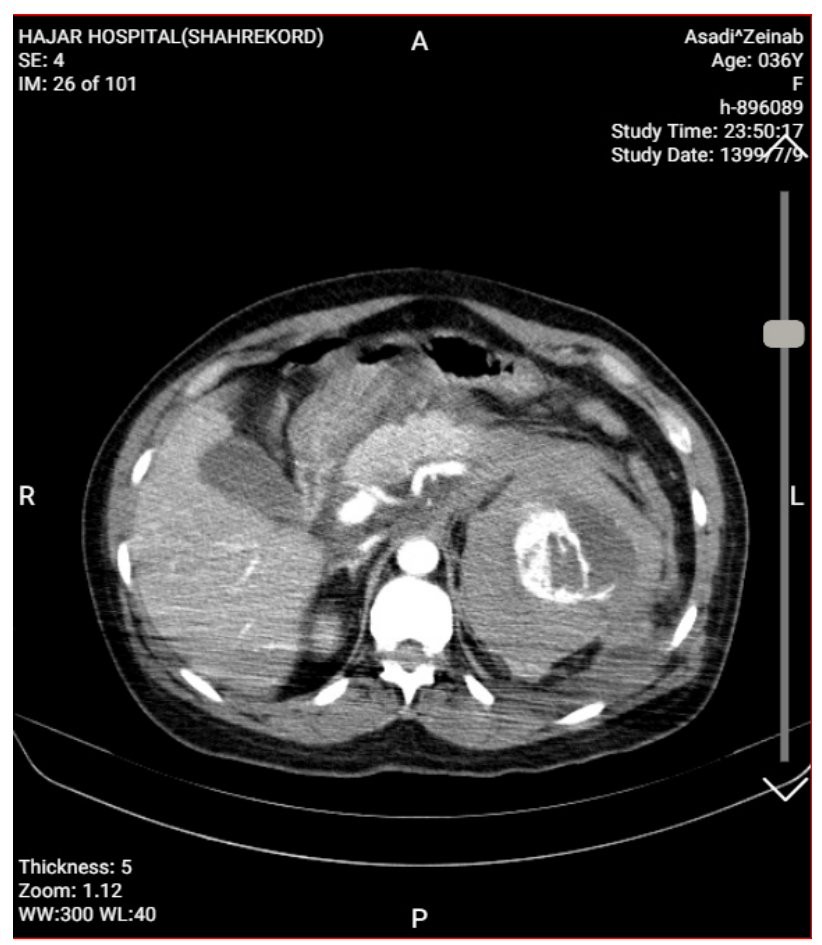

Figure 2. CT scan showing hemorrhagic renal cysts in the left kidney. Note. CT: Computerized tomography

gestation. In such conditions, in which accurate diagnosis could not be made by ultrasound, kidney cysts could be diagnosed in pregnancy with magnetic resonance imaging (MRI). Moreover, the pregnant woman underwent a cesarean section because of her emergency conditions and reduced FHR. Simple renal cysts are the most common cystic lesions found in adults and may be symptomatic at any time (3). Cysts are incidentally discovered in children and adults by ultrasonography, CT scan, or urography (4),

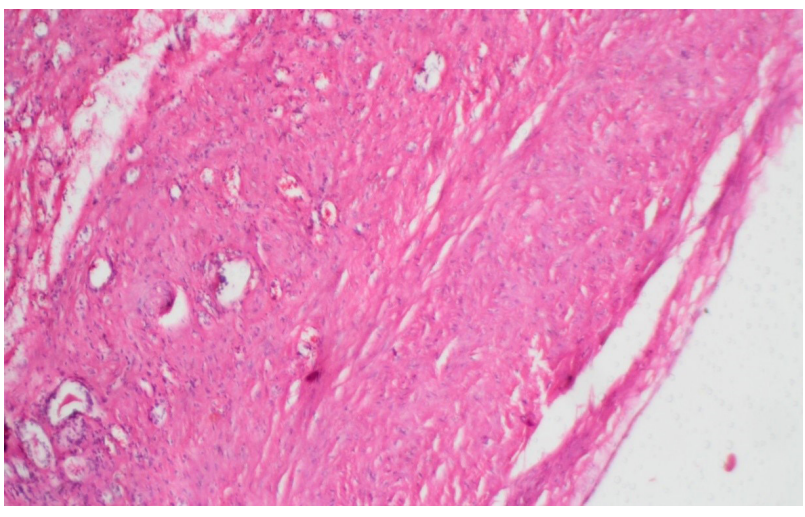

Figure 3. Pathological examination of a renal cyst.

but can be formed after abdominal mass pain, hematuria secondary to rupture into the pyelocalyceal system, segmental ischemia, and following spontaneous perinatal hemorrhage (SPH) in rare cases (5).

Wunderlich (1865) first described the clinical picture of SPH (6). The acute onset of abdominal and flank pain without trauma is the most common clinical presentation, and other presentations include hypovolemic shock, palpable mass, fever, anemia, and hematuria (7).

$\mathrm{SPH}$ is clinically a rare but potentially life-threatening condition, and clinicians need to diagnose and know its cause for favorable and proper treatment (8).

Perirenal hematoma commonly arises from a traumatic renal injury and rarely develops due to SPH (9). In a metaanalysis, Zhang et al (10) reported that the most important reasons for SPH were benign or malignant neoplasm (61\% of 101 cases), followed by renal cell carcinoma. The second etiological factor was vascular disease (17\%) with polyarteritis nodosa. Case report studies indicated that the causes of SPH were some pharmacological agents such as bevacizumab (11) and cocaine (12), autosomal dominant polycystic kidney disease, acquired cystic kidney disease, simple cysts, and preeclampsia (13). During pregnancy, the renal causes of non-traumatic retroperitoneal hematoma are associated with spontaneous rupture of the kidney or the existing lesions. Spontaneous renal rupture affects the collecting system or the kidney parenchyma, but rupture and bleeding occur in the renal pelvis or the calyceal system in the majority of patients. Hematologic diseases in pregnancy that lead to bleeding consist of platelet disorders, thrombotic microangiopathies such as thrombotic thrombocytopenic purpura, hemolysis, elevated liver enzyme levels, low platelet count (HELLP), and disseminated intravascular coagulation. Inherited clotting disorders include Von Willebrand disease, hemophilia A and hemophilia B, and factor X deficiency (14). On the other hand, preeclampsia can predispose pregnant patients to SPH. Although simple renal cysts and subsequent bleeding are rare, there is a need to carefully manage to avoid life-threatening complications (15).

This patient had a history of a simple left kidney cyst. The size of the cyst was $5 \mathrm{~cm}$ on ultrasound at the beginning of pregnancy. SPH was detected by contrastenhanced CT scan after the cesarean section rather than 
ultrasound, and then the patient underwent laparotomy again and the bleeding cyst was removed with partial nephrectomy. The pathological examination of the sample confirmed the diagnosis of a simple renal cyst.

\section{Conclusion}

In general, simple renal cysts should be considered as a rare cause of SPH in pregnancy. The diagnosis and treatment of SPH in pregnancy are also considered as critical challenges. An accurate diagnosis should be made by CT scan or MRI. Angioembolization is contraindicated in pregnancy, thus conservative treatment should be continued if the patient's hemodynamics are stable, otherwise, with the presence of a gynecologic surgical team, nephrectomy or partial nephrectomy can be performed as a preferable treatment.

\section{Conflict of Interests}

The authors declare no conflict of interests.

\section{Ethical Approval}

This report was published after obtaining the patient's consent. The protocol of this case report was approved by the Ethics Committee of Shahrekord University of Medical Sciences (code: IR.SKUMS. REC.1400.146).

\section{Acknowledgments}

This article was derived from a research project approved at Shahrekord University of Medical Sciences.

\section{Authors' Contributions}

FM and SA designed the study. SA diagnosed the disease and treated the patient. SA and FM drafted the manuscript, and both authors read and approved the final manuscript.

\section{Funding/Support}

None.

\section{References}

1. Partin AW, Wein AJ, Kavoussi LR, Peters CA, Dmochowski RR. Campbell Walsh Wein Urology, E-Book. Elsevier Health Sciences; 2020.

2. Blakeley CJ, Thiagalingham N. Spontaneous retroperitoneal haemorrhage from a renal cyst: an unusual cause of haemorrhagic shock. Emerg Med J. 2003;20(4):388. doi: 10.1136/emj.20.4.388.

3. Ng J, Loukogeorgakis S, Sanna E, Derwig I, Yu C, Paramasivam $\mathrm{G}$, et al. Postnatal outcome of prenatally-detected "simple" renal cysts: are they really simple? Early Hum Dev. 2021;157:105382. doi: 10.1016/j.earlhumdev.2021.105382.

4. Solinas A, Cau L, Fanari M, Flaviani I, Manca F, Melis M. Giant hydronephrosis secondary to ureterocele with duplex system in adults: report of a case. Arch Ital Urol Androl. 2020;92(4). doi: 10.4081/aiua.2020.4.318.

5. Choi HI, Kim YG, Kim SY, Jeong DW, Kim KP, Jeong KH, et al. Bilateral spontaneous perirenal hemorrhage due to initial presentation of polyarteritis nodosa. Case Rep Med. 2015;2015:428074. doi: 10.1155/2015/428074.

6. Daskalopoulos G, Karyotis I, Heretis I, Anezinis P, Mavromanolakis E, Delakas D. Spontaneous perirenal hemorrhage: a 10-year experience at our institution. Int Urol Nephrol. 2004;36(1):15-9. doi: 10.1023/b:urol.0000032680. 65742.9a.

7. Bittencourt PL, Cançado EL, Couto CA, Levy C, Porta G, Silva $A E$, et al. Brazilian society of hepatology recommendations for the diagnosis and management of autoimmune diseases of the liver. Arq Gastroenterol. 2015;52 Suppl 1:15-46. doi: 10.1590/s0004-28032015000500002.

8. Porcari GS, Jordan LC, Ichord RN, Licht DJ, Smith SE, Beslow LA. Outcome trajectories after primary perinatal hemorrhagic stroke. Pediatr Neurol. 2020;105:41-7. doi: 10.1016/j. pediatrneurol.2019.11.019.

9. Hayashi H, Okamoto I, Nakagawa K. Perirenal hematoma associated with bevacizumab treatment. Invest New Drugs. 2012;30(2):808-9. doi: 10.1007/s10637-010-9545-9.

10. Zhang JQ, Fielding JR, Zou KH. Etiology of spontaneous perirenal hemorrhage: a meta-analysis. J Urol. 2002;167(4):1593-6. doi: 10.1097/00005392-20020400000006.

11. Lee MS, Shin IS, Kwun DH, Kim SH, Kim HJ, Kim CK, et al. Perirenal hematoma in a patient treated with bevacizumab for metastatic colon cancer: a case report. Oncol Lett. 2016;11(5):3210-2. doi: 10.3892/ol.2016.4328.

12. Nzerue $C M$, Carew $K$, Faulkner $M$, Torrente $S$, Fleming $M$. Spontaneous perinephric haemorrhage and acute renal failure in pregnancy due to cocaine intoxication. NDT Plus. 2010;3(3):265-8. doi: 10.1093/ndtplus/sfq015.

13. Solazzo A, Testa F, Giovanella S, Busutti M, Furci L, Carrera P, et al. The prevalence of autosomal dominant polycystic kidney disease (ADPKD): A meta-analysis of European literature and prevalence evaluation in the Italian province of Modena suggest that ADPKD is a rare and underdiagnosed condition. PLoS One. 2018;13(1):e0190430. doi: 10.1371/journal. pone.0190430.

14. Strong J. Bleeding disorders in pregnancy. Curr Obstet Gynaecol. 2003;13(1):1-6. doi: 10.1054/cuog.2003.0300.

15. Esposito C, Soria-Gondek A, Castagnetti M, Cerulo M, Del Conte F, Esposito G, et al. Laparoscopic or robotic deroofing guided by indocyanine green fluorescence and perirenal fat tissue wadding technique of pediatric simple renal cysts. J Laparoendosc Adv Surg Tech A. 2020;30(4):471-6. doi: 10.1089/lap.2019.0650. 